Honam Mathematical J. 35 (2013), No. 2, pp. 311-317

http://dx.doi.org/10.5831/HMJ.2013.35.2.311

\title{
HYPONORMALITY OF TOEPLITZ OPERATORS ON THE WEIGHTED BERGMAN SPACES
}

\author{
Jongrak LeE AND Youho LeE*
}

\begin{abstract}
In this note we consider the hyponormality of Toeplitz operators $T_{\varphi}$ on the Weighted Bergman space $A_{\alpha}^{2}(\mathbb{D})$ with symbol in the class of functions $f+\bar{g}$ with polynomials $f$ and $g$ of degree 2 .
\end{abstract}

\section{Introduction}

A bounded linear operator $A$ on a Hilbert space is said to be hyponormal if its selfcommutator $\left[A^{*}, A\right]:=A^{*} A-A A^{*}$ is positive (semidefinite). Let $\mathbb{D}$ be the open unit disk in the complex plane. For $-1<\alpha<\infty$, the weighted Bergman space $A_{\alpha}^{2}(\mathbb{D})$ of the unit disk $\mathbb{D}$ is the space of analytic functions in $L^{2}\left(\mathbb{D}, d A_{\alpha}\right)$, where

$$
d A_{\alpha}(z)=(\alpha+1)\left(1-|z|^{2}\right)^{\alpha} d A(z) .
$$

The space $L^{2}\left(\mathbb{D}, d A_{\alpha}\right)$ is a Hilbert space with the inner product

$$
\langle f, g\rangle_{\alpha}=\int_{\mathbb{D}} f(z) \overline{g(z)} d A_{\alpha}(z) \quad\left(f, g \in L^{2}\left(\mathbb{D}, d A_{\alpha}\right)\right) .
$$

If $\alpha=0$ then $A_{0}^{2}(\mathbb{D})$ is the Bergman space $A^{2}(\mathbb{D})$. For any nonnegative integer $n$, let

$$
e_{n}(z)=\sqrt{\frac{\Gamma(n+\alpha+2)}{\Gamma(n+1) \Gamma(\alpha+2)}} z^{n} \quad(z \in \mathbb{D})
$$

where $\Gamma(s)$ stand for the usual Gamma function. It is easy to check that $\left\{e_{n}\right\}$ is an orthonormal basis for $A_{\alpha}^{2}(\mathbb{D})([10])$. For $\varphi \in L^{\infty}(\mathbb{D})$, the

Received April 25, 2013. Accepted May 20, 2013.

2010 Mathematics Subject Classification. Primary 47B20, 47B35.

Key words and phrases. Toeplitz operators, hyponormal, weighted Bergman space.

${ }^{*}$ Corresponding author 
Toeplitz operator $T_{\varphi}$ and the Hankel operator $H_{\varphi}$ on $A_{\alpha}^{2}(\mathbb{D})$ are defined by

$$
T_{\varphi} f:=P_{\alpha}(\varphi \cdot f) \quad \text { and } \quad H_{\varphi} f=\left(I-P_{\alpha}\right)(\varphi \cdot f) \quad\left(f \in A_{\alpha}^{2}(\mathbb{D}),\right.
$$

where $P_{\alpha}$ denotes the orthogonal projection that map from $L^{2}\left(\mathbb{D}, d A_{\alpha}\right)$ onto $A_{\alpha}^{2}(\mathbb{D})$. The reproducing kernel in $A_{\alpha}^{2}(\mathbb{D})$ is given by

$$
K_{z}^{(\alpha)}(\omega)=\frac{1}{(1-\bar{z} \omega)^{2+\alpha}},
$$

for $z, \omega \in \mathbb{D}$. We thus have

$$
\left(T_{\varphi} f\right)(z)=\int_{\mathbb{D}} \frac{\varphi(\omega) f(\omega)}{(1-z \bar{\omega})^{2+\alpha}} d A_{\alpha}(\omega),
$$

for $f \in A_{\alpha}^{2}(\mathbb{D})$ and $\omega \in \mathbb{D}$.

The hyponormality of Toeplitz operators on the Hardy space $H^{2}(\mathbb{T})$ of the unit circle $\mathbb{T}=\partial \mathbb{D}$ has been studied by C. Cowen [1], R.E. Curto, I.S. Hwang and W.Y. Lee [2], [3], [5] and others [4]. Recently, in [7], the hyponormality of $T_{\varphi}$ on the weighted Bergman space $A_{\alpha}^{2}(\mathbb{D})$ was studied. In [1], Cowen characterized the hyponormality of Toeplitz operator $T_{\varphi}$ on $H^{2}(\mathbb{T})$ by properties of the symbol $\varphi \in L^{\infty}(\mathbb{T})$. Here we shall employ an equivalent variant of cowen's theorem that was first proposed by T. Nakazi and K. Takahashi [8].

Cowen's Theorem ([1], [8]). For $\varphi \in L^{\infty}(\mathbb{T})$, write

$$
\mathcal{E}(\varphi):=\left\{k \in H^{\infty}:\|k\|_{\infty} \leq 1 \text { and } \varphi-k \bar{\varphi} \in H^{\infty}(\mathbb{T})\right\} .
$$

Then $T_{\varphi}$ is hyponormal if and only if $\mathcal{E}(\varphi)$ is nonempty.

The solution is based on a dilation theorem of Sarason [9]. For the weighted Bergman space, no dilation theorem (similar to Sarason's theorem) is available. In [6], the first named author characterized the hyponormality of $T_{\varphi}$ on $A_{\alpha}^{2}(\mathbb{D})$ in terms of the coefficients of the trigonometric polynomial $\varphi$ under certain assumptions about the coefficients of $\varphi$ on the weighted Bergman space when $\alpha \geq 0$.

Theorem A $([6])$. Let $\varphi(z)=\overline{g(z)}+f(z)$, where $f(z)=a_{1} z+a_{2} z^{2}$ and $g(z)=a_{-1} z+a_{-2} z^{2}$. If $a_{1} \overline{a_{2}}=a_{-1} \overline{a_{-2}}$ and $\alpha \geq 0$, then

$$
\begin{aligned}
& T_{\varphi} \text { on } A_{\alpha}^{2}(\mathbb{D}) \text { is hyponormal } \\
& \Longleftrightarrow \begin{cases}\frac{1}{\alpha+3}\left(\left|a_{2}\right|^{2}-\left|a_{-2}\right|^{2}\right) \geq \frac{1}{2}\left(\left|a_{-1}\right|^{2}-\left|a_{1}\right|^{2}\right) & \text { if }\left|a_{-2}\right| \leq\left|a_{2}\right| \\
4\left(\left|a_{-2}\right|^{2}-\left|a_{2}\right|^{2}\right) \leq\left|a_{1}\right|^{2}-\left|a_{-1}\right|^{2} & \text { if }\left|a_{2}\right| \leq\left|a_{-2}\right| .\end{cases}
\end{aligned}
$$


In this note we consider the hyponormality of Toeplitz operators $T_{\varphi}$ on $A_{\alpha}^{2}(\mathbb{D})$ with symbol in the class of functions $f+\bar{g}$ with polynomials $f$ and $g$ of degree 2. Since the hyponormality of operators is translation invariant we may assume that $f(0)=g(0)=0$. The following relations can be easily proved:

(1.1) $T_{\varphi+\psi}=T_{\varphi}+T_{\psi} \quad\left(\varphi, \psi \in L^{\infty}\right)$;

(1.2) $T_{\varphi}^{*}=T_{\bar{\varphi}} \quad\left(\varphi \in L^{\infty}\right)$

(1.3) $T_{\bar{\varphi}} T_{\psi}=T_{\bar{\varphi} \psi}$ if $\varphi$ or $\psi$ is analytic.

The purpose of this paper is to prove the Theorem A for the Toeplitz operators on $A_{\alpha}^{2}(\mathbb{D})$ when $-1<\alpha<0$.

\section{Main result.}

We need several auxiliary lemmas to prove Theorem 1 . We begin with:

Lemma 1. ([6]). For any $s, t$ nonnegative integers,

$$
P_{\alpha}\left(\bar{z}^{t} z^{s}\right)= \begin{cases}\frac{\Gamma(s+1) \Gamma(s-t+\alpha+2)}{\Gamma(s+\alpha+2) \Gamma(s-t+1)} z^{s-t} & \text { if } s \geq t \\ 0 & \text { if } s<t .\end{cases}
$$

Write

$$
k_{i}(z):=\sum_{n=0}^{\infty} c_{2 n+i} z^{2 n+i} \quad(i=0,1) .
$$

We then have:

Lemma 2. ([6]). For $0 \leq m \leq 2$ and $i=0,1$, we have

(i) $\left\|\bar{z}^{m} k_{i}(z)\right\|_{\alpha}^{2}=\sum_{n=0}^{\infty} \frac{(2 n+m+i) ! \Gamma(\alpha+2)}{\Gamma(2 n+m+i+\alpha+2)}\left|c_{2 n+i}\right|^{2}$

(ii) $\left\|P_{\alpha}\left(\bar{z}^{m} k_{i}(z)\right)\right\|_{\alpha}^{2}$

$$
= \begin{cases}\sum_{n=0}^{\infty} \frac{(2 n+i) !^{2} \Gamma(2 n+i-m+\alpha+2) \Gamma(\alpha+2)}{\Gamma(2 n+i+\alpha+2)^{2} \Gamma(2 n+i-m+1)}\left|c_{2 n+i}\right|^{2} & \text { if } m \leq i \\ \sum_{n=1}^{\infty} \frac{(2 n+i) !^{2} \Gamma(2 n+i-m+\alpha+2) \Gamma(\alpha+2)}{\Gamma(2 n+i+\alpha+2)^{2} \Gamma(2 n+i-m+1)}\left|c_{2 n+i}\right|^{2} & \text { if } m>i .\end{cases}
$$


We are ready for:

Theorem 1. Let $\varphi(z)=\overline{g(z)}+f(z)$, where $f(z)=a_{1} z+a_{2} z^{2}$ and $g(z)=a_{-1} z+a_{-2} z^{2}$. If $a_{1} \overline{a_{2}}=a_{-1} \overline{a_{-2}}$ and $-1<\alpha<\infty$, then

$T_{\varphi}$ on $A_{\alpha}^{2}(\mathbb{D})$ is hyponormal

$\Longleftrightarrow \begin{cases}\frac{1}{\alpha+3}\left(\left|a_{2}\right|^{2}-\left|a_{-2}\right|^{2}\right) \geq \frac{1}{2}\left(\left|a_{-1}\right|^{2}-\left|a_{1}\right|^{2}\right) & \text { if }\left|a_{-2}\right| \leq\left|a_{2}\right| \\ 4\left(\left|a_{-2}\right|^{2}-\left|a_{2}\right|^{2}\right) \leq\left|a_{1}\right|^{2}-\left|a_{-1}\right|^{2} & \text { if }\left|a_{2}\right| \leq\left|a_{-2}\right| .\end{cases}$

Proof. By Theorem A, we may assume that $-1<\alpha<0$. For $i=0,1$, put

$$
K_{i}:=\left\{k_{i}(z) \in A_{\alpha}^{2}(\mathbb{D}): k_{i}(z)=\sum_{n=0}^{\infty} c_{2 n+i} z^{2 n+i}\right\} .
$$

Then a straightforward calculation shows that $T_{\varphi}$ is hyponormal if and only if

$$
\begin{gathered}
\left\langle\left(H_{\bar{f}}^{*} H_{\bar{f}}-H_{\bar{g}}^{*} H_{\bar{g}}\right)\left(k_{0}(z)+k_{1}(z)\right),\left(k_{0}(z)+k_{1}(z)\right)\right\rangle_{\alpha} \geq 0 \\
\text { for all } k_{i} \in K_{i}(i=0,1) .
\end{gathered}
$$

Also we have that

$$
\begin{aligned}
& \left\langle H_{\bar{f}}^{*} H_{\bar{f}}\left(k_{0}(z)+k_{1}(z)\right),\left(k_{0}(z)+k_{1}(z)\right)\right\rangle_{\alpha} \\
& =\left\langle H_{\bar{f}} k_{0}(z), H_{\bar{f}} k_{0}(z)\right\rangle_{\alpha}+\left\langle H_{\bar{f}} k_{0}(z), H_{\bar{f}} k_{1}(z)\right\rangle_{\alpha} \\
& +\left\langle H_{\bar{f}} k_{1}(z), H_{\bar{f}} k_{0}(z)\right\rangle_{\alpha}+\left\langle H_{\bar{f}} k_{1}(z), H_{\bar{f}} k_{1}(z)\right\rangle_{\alpha},
\end{aligned}
$$

and

$$
\begin{aligned}
& \left\langle H_{\bar{g}}^{*} H_{\bar{g}}\left(k_{0}(z)+k_{1}(z)\right),\left(k_{0}(z)+k_{1}(z)\right)\right\rangle_{\alpha} \\
& =\left\langle H_{\bar{g}} k_{0}(z), H_{\bar{g}} k_{0}(z)\right\rangle_{\alpha}+\left\langle H_{\bar{g}} k_{0}(z), H_{\bar{g}} k_{1}(z)\right\rangle_{\alpha} \\
& +\left\langle H_{\bar{g}} k_{1}(z), H_{\bar{g}} k_{0}(z)\right\rangle_{\alpha}+\left\langle H_{\bar{g}} k_{1}(z), H_{\bar{g}} k_{1}(z)\right\rangle_{\alpha} .
\end{aligned}
$$

Substituting (2.2) and (2.3) into (2.1), it follows from Lemma 2 that

$$
\begin{aligned}
& T_{\varphi} \text { : hyponormal } \\
& \Longleftrightarrow\left\langle\left(H_{\bar{f}}^{*} H_{\bar{f}}-H_{\bar{g}}^{*} H_{\bar{g}}\right)\left(k_{0}(z)+k_{1}(z)\right),\left(k_{0}(z)+k_{1}(z)\right)\right\rangle_{\alpha} \geq 0 \\
& \Longleftrightarrow \sum_{i=0}^{1}\left(\left\|\bar{f} k_{i}\right\|_{\alpha}^{2}-\left\|\bar{g} k_{i}\right\|_{\alpha}^{2}+\left\|P_{\alpha}\left(\bar{g} k_{i}\right)\right\|_{\alpha}^{2}-\left\|P_{\alpha}\left(\bar{f} k_{i}\right)\right\|_{\alpha}^{2}\right) \geq 0 .
\end{aligned}
$$


Therefore it follows from Lemma 2 that $T_{\varphi}$ is hyponormal if and only if

$$
\begin{aligned}
& \left(\left|a_{1}\right|^{2}-\left|a_{-1}\right|^{2}\right)\left\{\frac{1}{\Gamma(\alpha+3)}\left|c_{0}\right|^{2}+\sum_{n=1}^{\infty}\left(\frac{\Gamma(2 n+2)}{\Gamma(2 n+\alpha+3)}\right.\right. \\
& \left.-\frac{(2 n)^{2} \Gamma(2 n)}{(2 n+\alpha+1)^{2} \Gamma(2 n+\alpha+1)}\right)\left|c_{2 n}\right|^{2}+\sum_{n=0}^{\infty}\left(\frac{\Gamma(2 n+3)}{\Gamma(2 n+\alpha+4)}\right. \\
& \left.\left.-\frac{(2 n+1)^{2} \Gamma(2 n+1)}{(2 n+\alpha+2)^{2} \Gamma(2 n+\alpha+2)}\right)\left|c_{2 n+1}\right|^{2}\right\}+\left(\left|a_{2}\right|^{2}-\left|a_{-2}\right|^{2}\right)\left\{\frac{2}{\Gamma(\alpha+4)}\left|c_{0}\right|^{2}\right. \\
& +\sum_{n=1}^{\infty}\left(\frac{\Gamma(2 n+3)}{\Gamma(2 n+\alpha+4)}-\frac{(2 n-1)^{2}(2 n)^{2} \Gamma(2 n-1)}{(2 n+\alpha+1)^{2}(2 n+\alpha)^{2} \Gamma(2 n+\alpha)}\right)\left|c_{2 n}\right|^{2} \\
& \left.+\frac{6}{\Gamma(\alpha+5)}\left|c_{1}\right|^{2}+\sum_{n=1}^{\infty}\left(\frac{\Gamma(2 n+4)}{\Gamma(2 n+\alpha+5)}\right)\left|c_{2 n+1}\right|^{2}\right\} \geq 0,
\end{aligned}
$$

or equivalently

$$
\begin{aligned}
& \left(\left|a_{1}\right|^{2}-\left|a_{-1}\right|^{2}\right)\left\{\frac{1}{\Gamma(\alpha+3)}\left|c_{0}\right|^{2}+\sum_{n=1}^{\infty}\left(\frac{\Gamma(n+2)}{\Gamma(n+\alpha+3)}\right.\right. \\
& \left.\left.-\frac{n^{2} \Gamma(n)}{(n+\alpha+1)^{2} \Gamma(n+\alpha+1)}\right)\left|c_{n}\right|^{2}\right\}+\left(\left|a_{2}\right|^{2}-\left|a_{-2}\right|^{2}\right) \\
& \times\left\{\sum_{n=0}^{1} \frac{\Gamma(n+3)}{\Gamma(n+\alpha+4)}\left|c_{n}\right|^{2}+\sum_{n=2}^{\infty}\left(\frac{\Gamma(n+3)}{\Gamma(n+\alpha+4)}\right.\right. \\
& \left.\left.-\frac{(n-1)^{2} n^{2} \Gamma(n-1)}{(n+\alpha)^{2}(n+\alpha+1)^{2} \Gamma(n+\alpha)}\right)\left|c_{n}\right|^{2}\right\} \geq 0 .
\end{aligned}
$$

For $n \in \mathbb{N}$, define $\zeta_{\alpha}$ by

$$
\zeta_{\alpha}(n):=\frac{\frac{\Gamma(n+2)}{\Gamma(n+\alpha+3)}-\frac{n^{2} \Gamma(n)}{(n+\alpha+1)^{2} \Gamma(n+\alpha+1)}}{\frac{\Gamma(n+3)}{\Gamma(n+\alpha+4)}-\frac{(n-1)^{2} n^{2} \Gamma(n-1)}{(n+\alpha)^{2}(n+\alpha+1)^{2} \Gamma(n+\alpha)}} .
$$

A direct calculation gives that

$$
\zeta_{\alpha}(n)=\frac{(n+\alpha)(n+\alpha+1)(\alpha+1)}{4 n^{2}+4(\alpha+2) n+2 \alpha} .
$$


Write

$$
\zeta_{\alpha}(x):=\frac{(x+\alpha)(x+\alpha+1)}{4 x^{2}+4(\alpha+2) x+2 \alpha} \quad\left(x \in \mathbb{R}^{+}\right) .
$$

Then we have that

$$
\zeta_{\alpha}^{\prime}(x)=-\frac{(\alpha+1) x^{2}+\left(2 \alpha^{2}+5 \alpha\right) x+\left(\alpha^{3}+4 \alpha^{2}+\frac{9}{2} \alpha\right)}{\left(2 x^{2}+2(\alpha+2) x+\alpha\right)^{2}},
$$

which implies that $\zeta_{\alpha}(x)$ is strictly decreasing function when $\alpha \geq 0$. But $\zeta_{\alpha}(x)$ has a relative maximum $x=\frac{-2 \alpha^{2}-5 \alpha+\sqrt{-9 \alpha^{2}-18 \alpha}}{2(\alpha+1)}$ when $-1<\alpha<$ 0 . Put $k_{\alpha}:=\frac{-2 \alpha^{2}-5 \alpha+\sqrt{-9 \alpha^{2}-18 \alpha}}{2(\alpha+1)}$, then the maximum value of $\zeta_{\alpha}(n)$ is $\max \left\{\zeta_{\alpha}\left(\left[k_{\alpha}\right]\right), \zeta_{\alpha}\left(\left[k_{\alpha}\right]+1\right)\right\}$. If $0<k_{\alpha}<1$, then

$$
\max \left\{\zeta_{\alpha}(0), \zeta_{\alpha}(1)\right\}=\max \left\{\frac{\alpha+3}{2}, \frac{(\alpha+1)(\alpha+4)}{8 \alpha+12}\right\}=\frac{\alpha+3}{2} .
$$

Let $k_{\alpha} \geq 1$. Since $\zeta_{\alpha}\left(k_{\alpha}\right)$ is relative maximum,

$$
\zeta_{\alpha}\left(k_{\alpha}\right) \geq \max \left\{\zeta_{\alpha}\left(\left[k_{\alpha}\right]\right), \zeta_{\alpha}\left(\left[k_{\alpha}\right]+1\right)\right\} .
$$

It follows from $-1<\alpha<0$ that

$$
k_{\alpha}^{2}+k_{\alpha}-\alpha^{2}-2 \alpha \geq 0 .
$$

Therefore

$$
\zeta_{\alpha}\left(k_{\alpha}\right)=\frac{\left(k_{\alpha}+\alpha\right)\left(k_{\alpha}+\alpha+3\right)}{4 k_{\alpha}^{2}+4(\alpha+2) k_{\alpha}+2 \alpha} \leq \frac{1}{2} \leq \frac{\alpha+3}{2} .
$$

Hence

$$
\max \left\{\zeta_{\alpha}\left(\left[k_{\alpha}\right]\right), \zeta_{\alpha}\left(\left[k_{\alpha}\right]+1\right)\right\} \leq \frac{\alpha+3}{2} .
$$

Let $\left|a_{-2}\right| \leq\left|a_{2}\right|$ and hence $\left|a_{1}\right| \leq\left|a_{-1}\right|$. Observe that

$$
\lim _{n \rightarrow \infty} \zeta_{\alpha}(n)=\frac{1}{4}
$$

and

$$
\frac{\alpha+3}{2} \geq \frac{(\alpha+1)(\alpha+4)}{6(\alpha+2)} .
$$

Therefore $(2.4),(2.5)$ and $(2.7)$ give that $T_{\varphi}$ is hyponormal if and only if

$$
2\left(\left|a_{2}\right|^{2}-\left|a_{-2}\right|^{2}\right) \geq(\alpha+3)\left(\left|a_{-1}\right|^{2}-\left|a_{1}\right|^{2}\right) .
$$

Let $\left|a_{2}\right| \leq\left|a_{-2}\right|$ and hence $\left|a_{-1}\right| \leq\left|a_{1}\right|$. Since $\zeta_{\alpha}(n) \geq \frac{1}{4}$, it follows from (2.4), (2.5), (2.6) and (2.7) that $T_{\varphi}$ is hyponormal if and only if

$$
4\left(\left|a_{-2}\right|^{2}-\left|a_{2}\right|^{2}\right) \leq\left(\left|a_{1}\right|^{2}-\left|a_{-1}\right|^{2}\right) .
$$


This completes the proof.

\section{References}

[1] C. Cowen, Hyponormality of Toeplitz operators, Proc. Amer. Math. Soc. 103 (1988), 809-812.

[2] R.E. Curto, I.S. Hwang and W.Y. Lee, Hyponormality and subnormality of block Toeplitz operators, Adv. Math. 230 (2012), 2094-2151.

[3] R.E. Curto and W.Y. Lee, Joint hyponormality of Toeplitz pairs, Memoirs Amer. Math. Soc. 150(712) (2001).

[4] I.S. Hwang, I.H. Kim and W.Y. Lee, Hyponormality of Toeplitz operators with polynomial symbol, Math. Ann 313 (1999), 247-261.

[5] I.S. Hwang and W.Y. Lee, Hyponormality of trigonometric Toeplitz operators, Trans. Amer. Math. 354 (2002), 2461-2474.

[6] I.S. Hwang and J.R. Lee, Hyponormal Toeplitz operators on the weighted Bergman spaces, Math. Ineq. and App. 15 (2012), 323-330.

[7] Yufeng Lu and Chaomei Liu, Commutativity and hyponormality of Toeplitz operators on the weighted Bergman space, J. Korean Math. Soc. 46 (2009), 621-642.

[8] T. Nakazi and K. Takahashi, Hyponormal Toeplitz operators and extremal problems of Hardy spaces, Trans. Amer. Math. Soc. 338 (1993), 753-769.

[9] D. Sarason, Generalized interpolation in $H^{\infty}$, Trans. Amer. Math. Soc. 127 (1967), 179-203.

[10] K. Zhu, Hyponormal Toeplitz operators with polynomial symbols, Integral Equations and Operator Theory 21 (1995), 376-381.

Jongrak Lee

Department of Mathematics, Sungkyunkwan University,

Suwon 440-746, Korea.

E-mail: jjonglak@skku.edu

Youho Lee

Department of Internet Information, Daegu Haany University, Kyeongsan 712-715, Korea.

E-mail: youho@dhu.ac.kr 